\title{
Dangerous liaisons at the virological synapse
}

\author{
Vincent Piguet ${ }^{1}$ and Quentin Sattentau ${ }^{2}$
}

\author{
1Department of Dermatology and Venereology, University Hospital of Geneva, Geneva, Switzerland. 2The Sir William Dunn School of Pathology, \\ University of Oxford, Oxford, United Kingdom.
}

\begin{abstract}
Cell-to-cell viral transmission facilitates the propagation of HIV-1 and human T cell leukemia virus type 1. Mechanisms of cell-to-cell transmission by retroviruses were not well understood until the recent description of virological synapses (VSs). VSs function as specialized sites of immune cell-to-cell contact that direct virus infection. Deciphering the molecular mechanisms of VS formation provides a fascinating insight into how pathogens subvert immune cell communication programs and achieve viral spread.
\end{abstract}

In order for HIV-1 to gain a foothold within an uninfected host, small amounts of cell-free or cell-associated virus have to efficiently infect target cells at, or proximal to, the portal of entry into the body and subsequently disseminate throughout susceptible tissues. Three mechanisms of viral spread have been described for HIV-1 (Figure 1). The first, the "classical" route of entry, is characterized by the binding of cell-free virions to a permissive host cell via various potential receptor interactions, followed by entry into the cytoplasm by fusion and subsequent steps in the replicative process (1-3). Second, cells such as DCs can capture virus via viral binding to C-type lectins or other cell-surface receptors, without necessarily becoming infected, and re-present infectious virus to a permissive target cell (a process known as infection in trans [4-8]). Third, an HIV-1-infected cell can infect a second cell without the requirement for release of cell-free virions into the surrounding extracellular milieu, which represents viral propagation through direct cell-to-cell transmission.

Cell-to cell transmission favors HIV-1 replication because it obviates the rate-limiting step of virus diffusion prior to attachment and might reduce or prevent viral neutralization by antibodies and complement (ref. 9 and Q. Sattentau, unpublished observations). This mode of viral propagation is less well understood than cell-free virus infection. However, the recent description of virological synapses (VSs) that form between cells infected, or pulsed but remaining uninfected, with HIV-1 (known as effector cells) and uninfected, receptor-expressing target cells finally suggests a mechanism for cell-to-cell viral transmission (10-13). Since the movement of viruses and other intracellular pathogens into and between cells is generally well documented (reviewed in refs. 14-16), here we will restrict ourselves to discussing direct cell-to-cell transfer of retroviruses between cells of the immune system by the use of a VS.

\section{Neural, immunological, and virological synapses}

The word synapse is derived from Greek, meaning "point of contact" (syn: with; aptein: to join), and the term is widely used in the biomedical literature: the neural synapse, the immunological syn-

Nonstandard abbreviations used: CCR5, CC chemokine receptor 5; cSMAC, central supramolecular activation complex; CXCR4, CXC chemokine receptor 4; HTLV-1, human T cell leukemia virus type 1; IS, immunological synapse; LC, Langerhans cell; LFA-1, lymphocyte function-associated antigen 1; MTOC, microtubule-organizing center; PSMAC, peripheral supramolecular activation complex; SARS-CoV, SARS coronavirus; VS, virological synapse.

Conflict of interest: The authors have declared that no conflict of interest exists.

Citation for this article: J. Clin. Invest. 114:605-610 (2004).

doi:10.1172/JCI200422812. apse (IS) $(17,18)$, and now the VS (10). The neural synapse was first described as a distinct organizational entity for intracellular signaling over 100 years ago and consists of points of contiguity between neurons across which information (via neurotransmitters) is relayed. Stability between the interacting neurons is established and maintained by an adhesion molecule scaffold, composed mainly of cadherins (18).

The recognition of a structured molecular architecture at the interface between T cells and APCs, which was subsequently termed the IS, was first documented 20 years ago $(19,20)$. The use of the term IS was later generalized to include all intercellular junctions that provide a stable environment for a variety of immunological events such as signaling via receptor engagement and directed secretion of cytokines, lytic granules, and other mediators. Unlike the neural synapse, the IS must rapidly assemble and disassemble between cells that are moving within and between tissues in order to allow these required immunological events to occur. For example, a single effector cell may assemble and disassemble ISs with multiple target cells in a rapid sequence, as has been observed, for example, during CTL-mediated killing (21).

The VS satisfies the minimal criteria for justifying the use of the term synapse. First, cells contact each other but remain individual entities (i.e., unfused). Second, a stable adhesive junction forms between the two cells. Third, the secretion of viral material from the pre- to post-synaptic side of this junction is directed across the synapse by cellular machinery, which in the case of the VS involves the transfer of viral material, including the genome, from the effector cell to the target cell.

\section{Virological synapses: the mystery of cell-to-cell retroviral transmission solved?}

Although the cell-to-cell spread of viruses has been documented for many years $(14,22-25)$, the first detailed description of a VS was only recently reported for the transmission of human $\mathrm{T}$ cell leukemia virus type 1 (HTLV-1). The cell-free form of HTLV-1 is very inefficient at infecting $\mathrm{T}$ cells and is spread between, and within, individuals by cell-to-cell transfer from infected $\mathrm{T}$ lymphocytes (26). A longstanding unsolved mystery in HTLV-1 pathogenesis was why $\mathrm{CD}^{+} \mathrm{T}$ cells were the principal target cell type in vivo, when the receptor for HTLV-1 entry was expressed essentially ubiquitously on mammalian cells. Elucidation of the mechanisms by which cell-to-cell HTLV-1 dissemination occurs has shed light on questions such as this, allowing a significant step forward in our understanding of retroviral pathogenesis (10).

In the case of HIV-1 infection, 2 types of VSs have been recently described: DC-T cell $(11,13)$ and T cell-T cell $(12)$. It is likely how- 
A

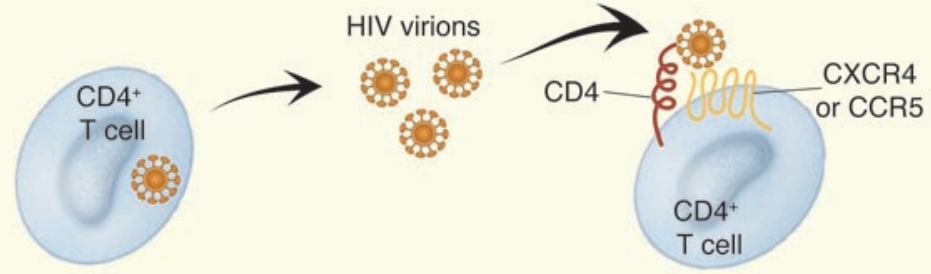

B

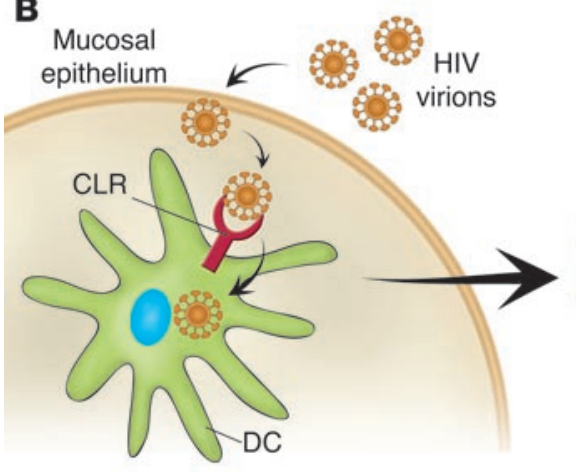

C

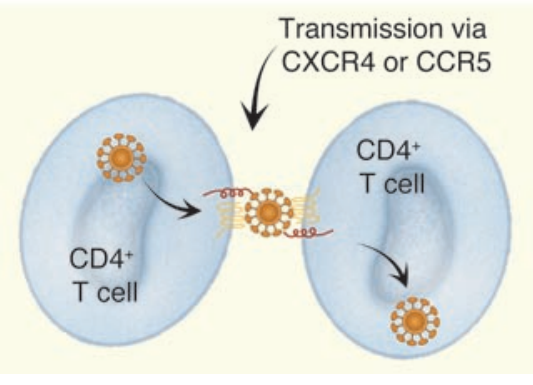

ever that viral transmission via the VS is not restricted to HTLV-1 and HIV-1 but is used by other intracellular viral pathogens in order to propagate. For instance, some preliminary evidence suggests that the formation of a VS might mediate the spread of the SARS coronavirus (SARS-CoV), since a SARS-CoV-pseudotyped lentiviral vector was shown to be transferred from DCs to target cells through a structure analogous to the HIV-1 VS (27). Other viruses that can infect or enter APCs may use similar strategies to hide from immune recognition and to facilitate their spread to permissive target cells.

\section{The VS in early HIV-1 infection: a role for the DC}

There are 3 model systems in which VSs have been described with regard to the sexual transmission of HIV-1. In the first system, the presynaptic cell is a DC or other APC that captures and internalizes incoming cell-free virions at the mucosa via interaction between cell-surface lectins (such as DC-SIGN) and the HIV-1 envelope glycoprotein, Env (Figure 2). The postsynaptic cell type studied in most detail to date is the $\mathrm{CD}^{+} \mathrm{T}$ cell (11), but VS assembly and function may not be limited to this target cell type in that any cell expressing the appropriate receptors may theoretically be infected via VS formation. The second model concerns the introduction of an infected $\mathrm{T}$ cell into the mucosa of an uninfected individual and subsequent spread from this $\mathrm{T}$ cell to another permissive cell type. Thus, using in vitro culture systems, HIV-1 has been shown to be transmitted directly from infected $\mathrm{T}$ cells to macrophages and,

\section{Figure 1}

Three mechanisms of HIV propagation. (A) Cellfree viral transmission. The classical route of viral propagation occurs via the binding of cellfree virions to a permissive host cell via CD4 and viral coreceptors (CCR5 or CXCR4), followed by viral entry into the cytoplasm by fusion and subsequent viral replication. (B) DC-T cell viral transmission. In the mucosal epithelia, DCs capture HIV virions via viral binding to C-type lectin-related (CLR) molecules or other cell-surface receptors, without necessarily becoming infected, and re-present infectious virus to CD4+ $\mathrm{T}$ cells after migration to the lymph nodes. (C) T cell-T cell viral transmission. An HIV-1-infected $\mathrm{CD} 4^{+} \mathrm{T}$ cell infects a second $\mathrm{CD} 4^{+} \mathrm{T}$ cell without the requirement for release of cell-free virions into the surrounding extracellular milieu, which represents viral propagation through direct cellto-cell transmission.

intriguingly, to epithelial cells carrying HIV receptors alternative to $\mathrm{CD} 4$, such as galactosyl ceramide. However, whether cell-to-cell infection of epithelial cells by HIV-1 has any in vivo relevance remains to be seen (reviewed in refs. 28 ,29). Finally, a related phenomenon has been described in which an infected $\mathrm{T}$ cell engages an epithelial cell, and virus is transcytosed across the epithelial cell into the underlying subepithelial tissue without the epithelial cell becoming infected $(30,31)$. Whether virusinduced T cell-epithelial cell synapses are related to, or distinct from, VSs formed between immune cells remains to be seen.

The importance of cell-to-cell virus transfer through the VS is unlikely to be restricted to the sexual transmission of HIV-1 and may well play a more general role in viral replication and dissemination. Formation of the VS has been observed in vitro between uninfected $\mathrm{T}$ cells and T cells infected with both HIV-1 (12) and HTLV-1 (10) and may help to explain the rapid dissemination of these viruses within secondary lymphoid tissues in vivo. We will review here known examples of VS formation, describe the potential relationship between the VS and the IS, and discuss the importance of VS assembly and function to HIV-1 pathogenesis in vivo.

The term DC encompasses several cellular subpopulations such as Langerhans cells (LCs) and myeloid dermal DCs. DCs and LCs probably play an important role during the early events of HIV-1 infection, most notably during sexual transmission $(28,29,32-34)$. DCs reside in the skin and mucosal tissues in a resting, immature state until they encounter pathogen-associated antigens. Upon exposure to a variety of stimuli, including bacterial products (35), TNF family ligands $(36,37)$, and double-stranded (38) or single-stranded RNA (39), DCs become activated and differentiate into mature APCs (40-42). This maturation is closely linked to the migration of DCs from peripheral tissues to secondary lymphoid organs. Within lymphoid tissue, antigen-activated LCs and DCs interact with antigen-specific $\mathrm{T}$ cells to initiate immune responses (43-47). HIV-1 infects LCs and other types of myeloid DCs both in vivo and in vitro (48-51), although inefficiently compared with HIV-1 infection of $\mathrm{CD}^{+} \mathrm{T}$ cells. In addition to allowing a low level of viral replication, DCs can 

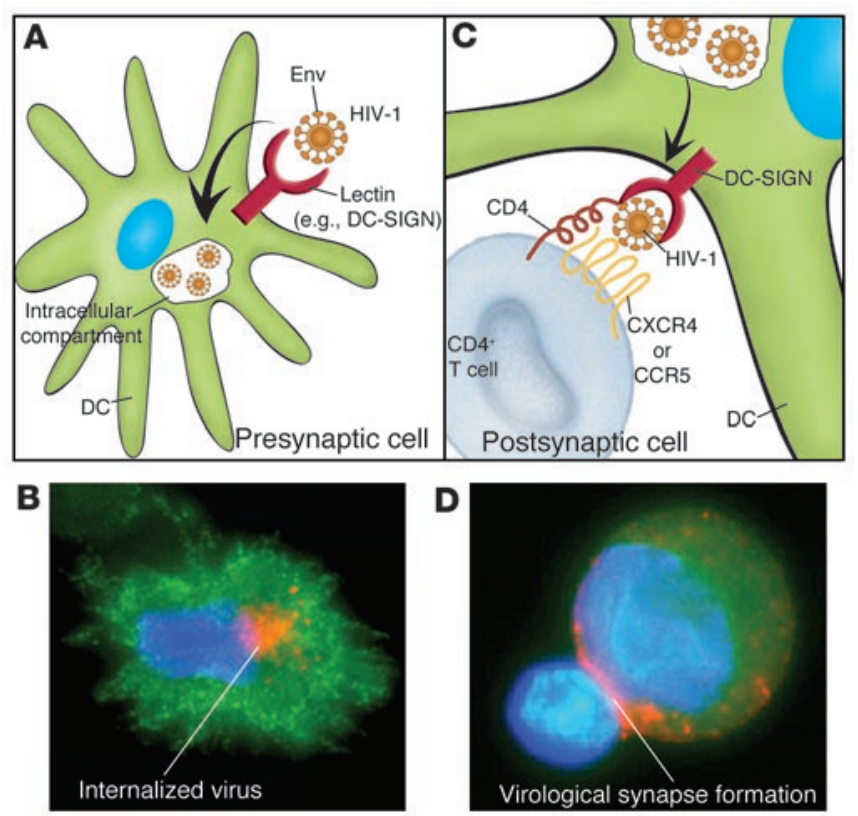

Figure 2

During DC-T cell viral transmission, rapid viral recycling to the DC-T cell zone of contact is observed. (A and B) HIV-1 is captured by C-type lectins (e.g., DC-SIGN) or other receptors and is internalized inside the DC. HIV-1 accumulates in intracellular compartments in DCs. (C and D) Upon encountering a CD4+ $\mathrm{T}$ cell, HIV-1 is rapidly redistributed from intracellular compartments to the zone of contact (the VS) between the $\mathrm{DC}$ and the $\mathrm{CD} 4^{+} \mathrm{T}$ cell. This leads to infection of the $\mathrm{CD} 4^{+} \mathrm{T}$ cell in trans and massive viral replication in DC-T cell clusters. Blue: DC nuclei; green: cytoplasm; orange: HIV-1 virions.

capture HIV-1 in an infectious form, without necessarily becoming infected themselves, and transfer viral material to $\mathrm{CD}^{+} \mathrm{T}$ cells, which results in vigorous infection (refs. 13, 52, and reviewed in refs. 29,32 , and 53). In immature DC subtypes, the C-type lectin DCSIGN (also known as CD209) is highly expressed and is the principal molecule mediating HIV-1 attachment and transfer to T cells in trans $(4,54)$. Other subsets of DCs, such as LCs, do not express detectable levels of DC-SIGN but are nevertheless able to capture and transfer HIV-1 infection via binding to other lectins such as the mannose receptor and langerin (6-8). In addition, adhesion molecules on DCs can recognize their cognate ligands (such as ICAM-1), which are incorporated into the membranes of HIV-1 virions during budding from infected cells (55). To date, DC-SIGN has been shown to function as an attachment factor for HIV-1, HIV-2, simian immunodeficiency virus (56,57), CMV (58), Dengue virus (59), Ebola virus (60), and SARS-CoV (27).

Interestingly, HIV-1 taken up via DC-SIGN remains infectious for prolonged periods of time (4). Although professional APCs such as DCs are rich in degradative compartments that are important in antigen processing, and some degradation of HIV-1 occurs, DCs appear unable to completely digest this virus $(13,61,62)$. Instead, DCs retain a population of infectious virus in an as-yet-unidentified intracellular compartment. DC-SIGN is an ICAM-3 receptor with a cytoplasmic domain containing well-defined endocytosis motifs (63). Not surprisingly therefore, HIV-1 bound to DC-SIGN has been shown to traffic through nonlysosomal endosomal compartments (61), some of which may have similarities to late endosomes
(V. Piguet, unpublished observations). This helps to explain why trypsin treatment of HIV-1-exposed DCs does not decrease the efficiency of DC-mediated virus transmission to T cells (64). In macrophages, HIV-1 buds into late endosomes using machinery implicated in the biogenesis of multivesicular bodies (mammalian class E vacuolar protein sorting proteins) and is subsequently released from cells by exocytosis (65-68). Whether HIV-1 trafficking within DCs intersects the same pathways is unknown. However, as mentioned above, both HIV-1-infected DCs and uninfected DCs carrying captured virus transmit robust infection to cocultured $\mathrm{CD}^{+} \mathrm{T}$ cells, although the relative contribution of each mode of transfer to $T$ cells remains to be quantified $(8,52,69,70)$. In conclusion, therefore, DC-T cell interactions, critical to the generation of immune responses, also provide rich microenvironments for HIV-1 dissemination and amplification of virus replication.

Aspects of the molecular basis of this phenomenon can be explained by the recent characterization of a VS that assembles between DCs carrying fluorescently tagged HIV-1 and uninfected $\mathrm{CD}^{+} \mathrm{T}$ cells (11). In this model system, DCs are not infected, and this type of synapse has been termed an infectious synapse (refs. 11, 13; Figure 2). This is clearly different from VSs formed between uninfected and infected immune cells, but for consistency throughout the present article, we will use the term VS for all synapses in which the effector cell, DC or T cell, is either infected or pulsed with virus. VS assembly is probably initiated by a normal cellular process in which DCs form transient contacts with $\mathrm{T}$ cells without the requirement for antigen specificity: this is proposed to be a kind of "scanning" by a $\mathrm{T}$ cell to enable potential recognition of a cognate peptide presented by the APC (71). In conjugates such as this, in which the DC has been pulsed with HIV-1, virions concentrate at the contact surface with the T cell, while the HIV-1 receptors $\mathrm{CD} 4$ and $\mathrm{CC}$ chemokine receptor 5 (CCR5) appear to be partially enriched on the T cell at the site of contact with the DC (ref. 11; Figure 2, C and D). The receptor recruitment and virus focusing occurring at the synapse therefore explain, at least in part, why DC transmission of HIV-1 to T cells is such an efficient process.

Much remains to be elucidated with regard to the DC-T cell VS. First, is sexual transmission truly facilitated by VS formation? To date, VS formation has been described mainly in DC-T cell conjugates: specifically between myeloid dermal DCs and T cells facilitating the transfer of nonreplicating HIV-1/SIV (refs. 11, 13; Figure 1). However, viral uptake and replication by LCs (in addition to myeloid DCs) occurs during HIV-1 sexual transmission, particularly for HIV-1 strains that bind to CCR5, since this is the predominant transmitted strain $(72,73)$. Whether HIV-1 transmission to T cells by LCs requires the formation of a VS is unknown. Second, what is the molecular basis of DC VS assembly? In this respect, there is a lack of studies on the selective interference of interactions occurring within the DC-T cell VS using for example: (a) receptor-blocking antibodies; (b) inhibitors of receptor function, cytoskeletal rearrangement, and signaling; or (c) RNA interference of receptor expression. Third, in which compartment is HIV-1 stored following uptake by DC-SIGN, and what prevents, at least in part, loss of viral infectivity in this compartment? Fourth, what triggers movement of the virus-containing compartment to the VS? It is clear that the DC-T cell VS has currently only been demonstrated in an in vitro model and is currently somewhat limited both in molecular detail and potential in vivo relevance. Although it would be premature to conclude that VS formation is necessary for dissemination and amplification of HIV-1 during the 
early stages of infection, the elucidation of these and other questions will no doubt enrich the fields of virology, cell biology, and immunology for some years to come.

\section{T cell-T cell synapses and retroviral replication}

That HIV-1-infected T cells could rapidly polarize viral receptors on uninfected $\mathrm{T}$ cells following cell-cell contact was established some time ago (74-76), but the molecular details were not well understood. We now know that interaction of the HIV-1 Env protein on an effector cell, with CD4 and CXC chemokine receptor 4 (CXCR4) expressed on naive T cells, is necessary (but perhaps not sufficient) to activate actin-dependent recruitment of the viral receptors and lymphocyte function-associated antigen 1 (LFA-1) into a supramolecular cluster at the site of cell-cell contact. Actinmediated movement of receptors appears to require both disassembly and reassembly of F-actin and motor protein-mediated gliding of actin filaments, as demonstrated by the use of inhibitors of these two processes (12). Although the molecular signaling cascades implicated in the formation of the T cell VS have not been elucidated for either the HIV-1 or the HTLV-1 VS, Env-receptor engagement appears central to this process, since the inhibition of these interactions at the HIV-1 VS prevents its assembly (12). Intrinsic to the definition of a synapse is that a stable adhesive junction forms between the $\mathrm{T}$ cells. Although this is only inferred from the clustering of LFA-1 and its actin-anchor protein, talin, at the site of cell-cell contact, and functional studies have not yet been carried out, it seems probable that LFA- 1 is ligated by ICAMs on the opposing cell, thus clamping the two cells together (1012). Electron microscopic analysis of VS junctions shows discrete regions of tightly opposed membrane, reminiscent of adhesive patches of plasma membrane in the IS (21), which are most likely formed by the interaction of adhesion molecules.

Secretion at the VS takes the form of virus transfer from the presynaptic to the postsynaptic cell. A tantalizing observation probably relevant to this event is the reorientation of the microtubule-organizing center (MTOC) in the infected cell proximal to the site of cell-cell contact (ref. 10 and Q. Sattentau, unpublished observations). This suggests that microtubules may be implicated in VS function, a concept supported by the finding that nocodazole, an inhibitor of microtubule polymerization, prevents VS assembly and viral transfer (ref. 10 and Q. Sattentau, unpublished observations). Such a result is consistent with the reported use by HIV-1 of microtubules for intracellular transport (77). Thus HIV-1 and HTLV-1 may "hijack"

Figure 3 with ligated adhesion molecules. the lymphocyte secretion machinery to send virus to the site of cellular contact in a manner analogous to directed cytotoxic granule secretion at the CTL IS $(21,78)$. The movement of HIV-1 across the VS then appears to take place by viral budding at or near the site of cell-cell contact followed, most likely, by virion fusion with the target cell plasma membrane (12) (Figure 2).

\section{Potential relationships between ISs and VSs}

ISs between CTLs and their target cells (78-80), NK cells and their target cells $(81,82)$, and T and B cells (17) have been described. It is unclear at present how closely the VS is related to the IS and whether retroviruses simply harness cellular machinery that preexists for IS assembly for their own benefit or extensively modify other cellular programs in a more sophisticated manner. Answering this question is complicated by the fact that at least two types of IS have been proposed to occur between DCs and T cells: antigen dependent and antigen independent $(71,83-86)$. The initial adhesion interactions that allow $\mathrm{T}$ cells to attach to other cells of the immune system prior to any specific antigen interaction may be shared by the IS and VS, since, for example, uninfected T cells will form transient conjugates with other uninfected $\mathrm{T}$ cells (12).
A The virological (T cell) synapse

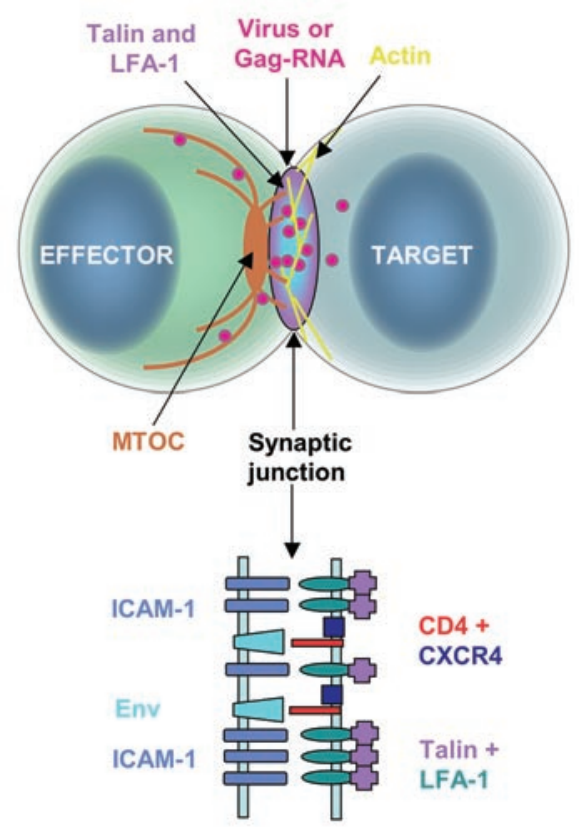

\section{B The immunological (CTL) synapse}
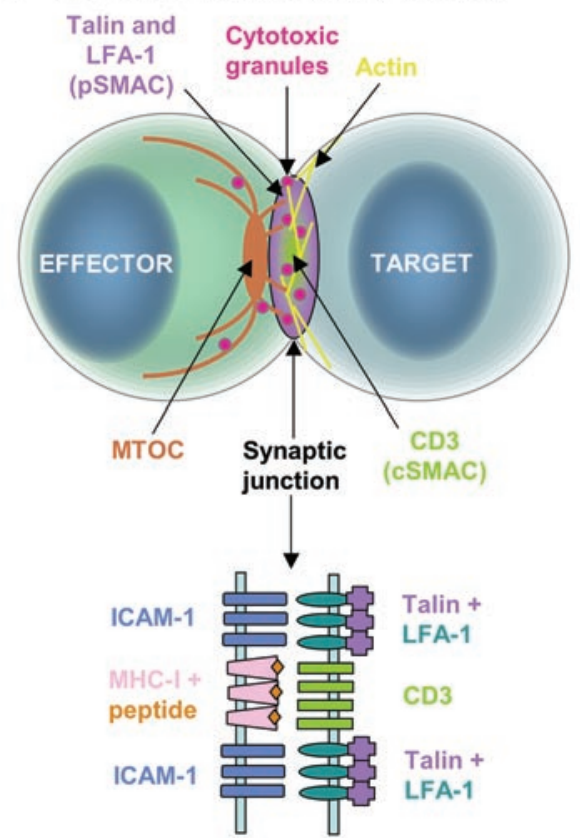

Similarities between the T cell-T cell VS and IS. The upper images of $\mathbf{A}$ and $\mathbf{B}$ show stylized features of synapses formed between T cells in the VS (A) and the IS (B). The VS schematic $(\mathbf{A})$ is based on a composite of known features of the HIV-1 and HTLV-1 T cell VSs and highlights features in common with, and divergent from, the IS. Within the VS, Env ligates CD4 and CXCR4 in the target cell and ICAM-1 engages LFA-1 in the target cell. Gag associated with the viral genome present within the effector cell is recruited along microtubules to the site of cell-cell contact and moves across the central zone of the synapse into the target cell. In the target cell, LFA-1-talin complexes and CD4 and CXCR4 are recruited in an actin-dependent manner to the VS. In the IS (B), LFA-1-talin complexes form the PSMAC, whereas the T cell receptor (TCR) is recruited to the cSMAC, into which cytotoxic granules are secreted in a microtubule-dependent manner. In the target cell, ICAM-1 engages LFA-1 and peptide-MHC-I complexes ligate the TCR of the effector cell. The lower parts of both panels illustrate a simplified schematic of the molecular arrangements present in the VS (A) and IS (B). The VS is shown in its partially disordered form with Env-receptor complexes interspersed 
Subsequent steps differ, however, at least in T cell-T cell VS assembly, since neither the HIV-1- nor the HTLV-1-induced VS appear to rely on $\mathrm{T}$ cell receptor engagement by cognate $\mathrm{MHC}$-peptide complexes on the effector cell, and there is no evidence for CD3 enrichment in the HIV-1 VS (12). Recognition of cognate MHCpeptide complexes leads to stabilization of the IS by formation of an integrin-containing peripheral supramolecular activation cluster (PSMAC), recruitment of additional T cell receptor molecules and PKC- $\theta$ into the central SMAC (cSMAC), and triggering of signaling events initiated by tyrosine phosphorylation and calcium flux (reviewed in refs. 84, 85; Figure 3). In the pSMAC, talin anchors LFA-1 into an adhesive "gasket" that engages with ICAMs on the effector cell. In the cSMAC, receptors are ligated, and soluble mediators are secreted across the synaptic cleft (21). This highly structured ringlike arrangement of the IS is absent in the HIV-1-induced T cell-T cell VS but may be at least partially present in the HTLV-1 VS. A talin ring has been observed in a percentage of HTLV-1 VSs with viral Gag protein localized in the central space, which suggests that virus may transfer, by an unknown mechanism, across a synaptic cleft depleted of adhesion interactions. A related observation is that, as described above, the MTOC realigns proximal to the site of cell-cell contact in the effector cell in both the IS and the HTLV-1- and HIV-1-induced VSs (ref. 10 and Q. Sattentau, unpublished observations). MTOC (and Golgi) orientation toward the synapse is an indication of directed secretion in the IS and may explain how viral Gag moves to the intercellular junction in the VS.

The rearrangement of the receptors CD3, CD4, and LFA-1 in the IS and CD4, CXCR4, and LFA-1 in the T cell VS is dependent upon actin remodeling. Similarly, inhibitors of microtubule function interfere with MTOC reorientation and the effector cell secretory apparatus in both types of synapse $(10,12)$ : disabling tubulin remodeling prevents Gag polarization to the VS and its subsequent transfer into the target cell (ref. 10 and Q. Sattentau, unpublished observations).

In the HIV-1-induced T cell-T cell VS, the engagement of receptors (CD4 and CXCR4) on the target cell by viral Env on the effector cell is central to VS assembly (Figure 1C), but signaling by integrins such as LFA-1 and other cell-surface receptors cannot be ruled out.
At present, the molecular details of how VS formation is triggered and the VS assembled remain to be elucidated, as does the process activating directed Gag targeting to the synaptic junction. There are clear similarities and differences between the IS and the VS, and likewise similarities and differences among the different VSs (e.g., HIV-1 and HTLV-1 T cell-T cell VSs and HIV-1 DC-T cell VS). Interestingly, the precise role of the IS in T cell activation is still being debated: for example it is unclear whether $\mathrm{T}$ cell signaling precedes the IS or takes place as a consequence of IS assembly (87). By contrast, the role of the VS appears clear: the virus subverts normal cellular processes in order to allow efficient and stealthy spread of infection between cells of the immune system.

\section{Conclusions}

The identification and partial characterization of the VS provides a mechanistic explanation for cell-to-cell retroviral infection. Although this phenomenon has so far been described only for two retroviruses (HIV-1 and HTLV-1), it is quite likely that other pathogenic viruses that infect cells of the immune system use related modes of spread. VSs share some common features with the IS but also exhibit important differences. Further study will help elucidate common and divergent pathways in the formation and function of these different types of cellular synapse and may point toward strategies for intervention in VS function as a novel therapeutic target.

\section{Acknowledgments}

We thank E. Garcia and J.F. Arrighi for providing images of DC-T cell VS formation and M. Marsh for helpful discussions during the preparation of this manuscript. This work was supported by Swiss National Science Foundation (SNF) grant 3345-67200.01 and funding provided by the Geneva Cancer League to V. Piguet; and MRC grant G0100137 and an EC FP6 consortium EMPRO grant to Q. Sattentau. V. Piguet is the recipient of an SNF professorship (PP00A - 68785).

Address correspondence to: Vincent Piguet, Department of Dermatology, HUG, 24 rue Micheli-du-Crest, 1211 Geneva, Switzerland. Phone: 41-22-372-94-65; Fax: 41-22-372-94-70; E-mail: vincent.piguet@medecine.unige.ch.
1. Pierson, T.C., and Doms, R.W. 2003. HIV-1 entry and its inhibition. Curr. Top. Microbiol. Immunol. 281:1-27.

2. Kilby, J.M., and Eron, J.J. 2003. Novel therapies based on mechanisms of HIV-1 cell entry. N. Engl. J. Med. 348:2228-2238.

3. Stebbing, J., Gazzard, B., and Douek, D.C. 2004 Where does HIV live? N. Engl. J. Med. 350:1872-1880.

4. Geijtenbeek, T.B., et al. 2000. DC-SIGN, a dendritic cell-specific HIV-1-binding protein that enhances trans-infection of T cells. Cell. 100:587-597.

5. Bobardt, M.D., et al. 2003. Syndecan captures, protects, and transmits HIV to T lymphocytes. Immunity. 18:27-39.

6. Nguyen, D.G., and Hildreth, J.E. 2003. Involvement of macrophage mannose receptor in the binding and transmission of HIV by macrophages. Eur. J. Immunol. 33:483-493.

7. Turville, S.G., et al. 2002. Diversity of receptors binding HIV on dendritic cell subsets. Nat. Immunol. 3:975-983.

8. Hu, Q., et al. 2004. Blockade of attachment and fusion receptors inhibits HIV-1 infection of human cervical tissue. J. Exp. Med. 199:1065-1075.

9. Ganesh, L., et al. 2004. Infection of specific dendritic cells by CCR5-tropic HIV-1 promotes cell-mediated transmission of virus resistant to broadly neutralizing antibodies. J. Virol. In press.

10. Igakura, T., et al. 2003. Spread of HTLV-I between lymphocytes by virus-induced polarization of the cytoskeleton. Science. 299:1713-1716.

11. McDonald, D., et al. 2003. Recruitment of HIV and its receptors to dendritic cell-T cell junctions. Science. 300:1295-1297.

12. Jolly, C., Kashefi, K., Hollinshead, M., and Sattentau, Q.J. 2004. HIV-1 cell to cell transfer across an Env-induced, actin-dependent synapse. J. Exp. Med. 199:283-293.

13. Turville, S.G., et al. 2004. Immunodeficiency virus uptake, turnover, and 2-phase transfer in human dendritic cells. Blood. 103:2170-2179.

14. Johnson, D.C., and Huber, M.T. 2002. Directed egress of animal viruses promotes cell-to-cell spread. J. Virol. 76:1-8.

15. Cossart, P., and Sansonetti, P.J. 2004. Bacterial invasion: the paradigms of enteroinvasive pathogens. Science. 304:242-248.

16. Sibley, L.D. 2004. Intracellular parasite invasion strategies. Science. 304:248-253.

17. Grakoui, A., et al. 1999. The immunological synapse: a molecular machine controlling $\mathrm{T}$ cell activation. Science. 285:221-227.
18. Dustin, M.L., and Colman, D.R. 2002. Neural and immunological synaptic relations. Science. 298:785-789.

19. Norcross, M.A. 1984. A synaptic basis for Tlymphocyte activation. Ann. Immunol. (Paris). 135D:113-134.

20. Paul, W.E., et al. 1987. Regulation of B-lymphocyte activation, proliferation, and differentiation. Ann. N. Y. Acad. Sci. 505:82-89.

21. Bossi, G., et al. 2002. The secretory synapse: the secrets of a serial killer. Immunol. Rev. 189:152-160.

22. Gupta, P., Balachandran, R., Ho, M., Enrico, A., and Rinaldo, C. 1989. Cell-to-cell transmission of human immunodeficiency virus type 1 in the presence of azidothymidine and neutralizing antibody. J. Virol. 63:2361-2365.

23. Sato, H., Orenstein, J., Dimitrov, D., and Martin, M. 1992. Cell-to-cell spread of HIV-1 occurs within minutes and may not involve the participation of virus particles. Virology. 186:712-724.

24. Phillips, D.M. 1994. The role of cell-to-cell transmission in HIV infection. AIDS. 8:719-731.

25. Carr, J.M., Hocking, H., Li, P., and Burrell, C.J. 1999. Rapid and efficient cell-to-cell transmission of human immunodeficiency virus infection from monocyte-derived macrophages to peripheral 
blood lymphocytes. Virology. 265:319-329.

26. Bangham, C.R. 2003. The immune control and cell-to-cell spread of human T-lymphotropic virus type 1. J. Gen. Virol. 84:3177-3189.

27. Yang, Z.Y., et al. 2004. pH-dependent entry of severe acute respiratory syndrome coronavirus is mediated by the spike glycoprotein and enhanced by dendritic cell transfer through DC-SIGN. J. Virol. 78:5642-5650.

28. Shattock, R.J., and Moore, J.P. 2003. Inhibiting sexual transmission of HIV-1 infection. Nat. Rev. Microbiol. 1:25-34.

29. Pope, M., and Haase, A.T. 2003. Transmission, acute HIV-1 infection and the quest for strategies to prevent infection. Nat. Med. 9:847-852.

30. Bomsel, M. 1997. Transcytosis of infectious human immunodeficiency virus across a tight human epithelial cell line barrier. Nat. Med. 3:42-47.

31. Bomsel, M., et al. 1998. Intracellular neutralization of HIV transcytosis across tight epithelial barriers by anti-HIV envelope protein IIgA or IgM. Immunity. 9:277-287.

32. Steinman, R.M., et al. 2003. The interaction of immunodeficiency viruses with dendritic cells. Curr. Top. Microbiol. Immunol. 276:1-30.

33. Turville, S., Wilkinson, J., Cameron, P., Dable, J., and Cunningham, A.L. 2003. The role of dendritic cell C-type lectin receptors in HIV pathogenesis. J. Leukoc. Biol. 74:710-718

34. Geijtenbeek, T.B., and van Kooyk, Y. 2003. DCSIGN: a novel HIV receptor on DCs that mediates HIV-1 transmission. Curr. Top. Microbiol. Immunol. 276:31-54.

35. Rescigno, M., Granucci, F., Citterio, S., Foti, M., and Ricciardi-Castagnoli, P. 1999. Coordinated events during bacteria-induced DC maturation. Immunol. Today. 20:200-203.

36. Caux, C., Dezutter-Dambuyant, C., Schmitt, D., and Banchereau, J. 1992. GM-CSF and TNFalpha cooperate in the generation of dendritic Langerhans cells. Nature. 360:258-261.

37. Rescigno, M., et al. 2000. Fas engagement induces the maturation of dendritic cells (DCs), the release of interleukin (IL)-1beta, and the production of interferon gamma in the absence of IL-12 during DC-T cell cognate interaction: a new role for Fas ligand in inflammatory responses. J. Exp. Med. 192:1661-1668.

38. Cella, M., Engering, A., Pinet, V., Pieters, J., and Lanzavecchia, A. 1997. Inflammatory stimuli induce accumulation of MHC class II complexes on dendritic cells. Nature. 388:782-787.

39. Heil, F., et al. 2004. Species-specific recognition of single-stranded RNA via toll-like receptor 7 and 8 . Science. 303:1526-1529.

40. Chow, A., Toomre, D., Garrett, W., and Mellman, I. 2002. Dendritic cell maturation triggers retrograde MHC class II transport from lysosomes to the plasma membrane. Nature. 418:988-994.

41. Boes, M., et al. 2002. T-cell engagement of dendritic cells rapidly rearranges MHC class II transport. Nature. 418:983-988.

42. Trombetta, E.S., Ebersold, M., Garrett, W., Pypaert, M., and Mellman, I. 2003. Activation of lysosomal function during dendritic cell maturation. Science. 299:1400-1403.

43. Banchereau, J., and Steinman, R.M. 1998. Dendritic cells and the control of immunity. Nature. 392:245-252.

44. Banchereau, J., et al. 2000. Immunobiology of dendritic cells. Annu. Rev. Immunol. 18:767-811.

45. Lanzavecchia, A., and Sallusto, F. 2001. Regulation of T cell immunity by dendritic cells. Cell. 106:263-266.

46. Stoll, S., Delon, J., Brotz, T.M., and Germain, R.N. 2002. Dynamic imaging of T cell-dendritic cell interactions in lymph nodes. Science. 296:1873-1876.
47. Mempel, T.R., Henrickson, S.E., and Von Andrian, U.H. 2004. T-cell priming by dendritic cells in lymph nodes occurs in three distinct phases. Nature. 427:154-159.

48. Tschachler, E., et al. 1987. Epidermal Langerhans cells - a target for HTLV-III/LAV infection. J. Invest. Dermatol. 88:233-237.

49. Ringler, D.J., et al. 1989. Cellular localization of simian immunodeficiency virus in lymphoid tissues. I. Immunohistochemistry and electron microscopy. Am. J. Pathol. 134:373-383.

50. Stahl-Hennig, C., et al. 1999. Rapid infection of oral mucosal-associated lymphoid tissue with simian immunodeficiency virus. Science. 285:1261-1265.

51. Hu, J., Gardner, M.B., and Miller, C.J. 2000. Simian immunodeficiency virus rapidly penetrates the cervicovaginal mucosa after intravaginal inoculation and infects intraepithelial dendritic cells. J. Virol. 74:6087-6095

52. Blauvelt, A., et al. 1997. Productive infection of dendritic cells by HIV- 1 and their ability to capture virus are mediated through separate pathways. J. Clin. Invest. 100:2043-2053.

53. Piguet, V., and Blauvelt, A. 2002. Essential roles for dendritic cells in the pathogenesis and potential treatment of HIV disease. J. Invest. Dermatol. 119:365-369

54. Arrighi, J.F., et al. 2004. Lentiviral-mediated RNA interference of DC-SIGN expression abrogates HIV transmission from dendritic cells to T cells. J. Virol. In press.

55. Tardif, M.R., and Tremblay, M.J. 2003. Presence of host ICAM-1 in human immunodeficiency virus type 1 virions increases productive infection of CD4+ T lymphocytes by favoring cytosolic delivery of viral material. J. Virol. 77:12299-12309.

56. Bashirova, A.A., et al. 2001. A dendritic cell-specific intercellular adhesion molecule 3-grabbing nonintegrin (DC-SIGN)-related protein is highly expressed on human liver sinusoidal endothelial cells and promotes HIV-1 infection. J. Exp. Med. 193:671-678

57. Pohlmann, S., et al. 2001. DC-SIGN interactions with human immunodeficiency virus: virus binding and transfer are dissociable functions. J. Virol. 75:10523-10526

58. Halary, F., et al. 2002. Human cytomegalovirus binding to DC-SIGN is required for dendritic cell infection and target cell trans-infection. Immunity. 17:653-664.

59. Tassaneetrithep, B., et al. 2003. DC-SIGN (CD209) mediates dengue virus infection of human dendritic cells. J. Exp. Med. 197:823-829.

60. Alvarez, C.P., et al. 2002. C-type lectins DC-SIGN and L-SIGN mediate cellular entry by Ebola virus in cis and in trans. J. Virol. 76:6841-6844.

61. Kwon, D.S., Gregorio, G., Bitton, N., Hendrickson, W.A., and Littman, D.R. 2002. DC-SIGN-mediated internalization of HIV is required for trans-enhancement of $\mathrm{T}$ cell infection. Immunity. 16:135-144.

62. Moris, A., et al. 2004. DC-SIGN promotes exogenous MHC-I-restricted HIV-1 antigen presentation. Blood. 103:2648-2654

63. Engering, A., et al. 2002. The dendritic cell-specific adhesion receptor DC-SIGN internalizes antigen for presentation to T cells. J. Immunol. 168:2118-2126.

64. Ayehunie, S., et al. 1995. Acutely infected Langerhans cells are more efficient than $\mathrm{T}$ cells in disseminating HIV type 1 to activated T cells following a short cell-cell contact. AIDS Res. Hum. Retroviruses. 11:877-884.

65. Garrus, J.E., et al. 2001. Tsg101 and the vacuolar protein sorting pathway are essential for HIV-1 budding. Cell. 107:55-65.

66. Martin-Serrano,J., Zang, T., and Bieniasz, P.D. 2001. HIV-1 and Ebola virus encode small peptide motifs that recruit Tsg101 to sites of particle assembly to facilitate egress. Nat. Med. 7:1313-1319.

67. Strack, B., Calistri, A., Craig, S., Popova, E., and Gottlinger, H.G. 2003. AIP1/ALIX is a binding partner for HIV-1 p6 and EIAV p9 functioning in virus budding. Cell. 114:689-699.

68. von Schwedler, U.K., et al. 2003. The protein network of HIV budding. Cell. 114:701-713.

69. Cameron, P.U., et al. 1992. Dendritic cells exposed to human immunodeficiency virus type- 1 transmit a vigorous cytopathic infection to CD4+ T cells. Science. 257:383-387.

70. Pope, M., et al. 1994. Conjugates of dendritic cells and memory $\mathrm{T}$ lymphocytes from skin facilitate productive infection with HIV-1. Cell. 78:389-398.

71. Revy, P., Sospedra, M., Barbour, B., and Trautmann, A. 2001. Functional antigen-independent synapses formed between $\mathrm{T}$ cells and dendritic cells. Nat. Immunol. 2:925-931.

72. Kawamura, T., et al. 2003. R5 HIV productively infects Langerhans cells, and infection levels are regulated by compound CCR5 polymorphisms. Proc. Natl. Acad. Sci.U. S. A. 100:8401-8406.

73. Sugaya, M., Lore, K., Koup, R.A., Douek, D.C. and Blauvelt, A. 2004. HIV-infected Langerhans cells preferentially transmit virus to proliferating autologous CD4(+) memory $\mathrm{T}$ cells located within Langerhans cell-T cell clusters. J. Immunol. 172:2219-2224

74. Phillips, D.M., and Bourinbaiar, A.S. 1992. Mechanism of HIV spread from lymphocytes to epithelia. Virology. 186:261-273.

75. Sattentau, Q.J., and Moore, J.P. 1993. The role of CD4 in HIV binding and entry. Pbilos. Trans. R. Soc. Lond. B Biol. Sci. 342:59-66.

76. Fais, S., et al. 1995. Unidirectional budding of HIV-1 at the site of cell-to-cell contact is associated with co-polarization of intercellular adhesion molecules and HIV-1 viral matrix protein. AIDS. 9:329-335.

77. McDonald, D., et al. 2002. Visualization of the intracellular behavior of HIV in living cells. J. Cell Biol. 159:441-452.

78. Stinchcombe, J.C., Bossi, G., Booth, S., and Griffiths, G.M. 2001. The immunological synapse of CTL contains a secretory domain and membrane bridges. Immunity. 15:751-761.

79. McGavern, D.B., Christen, U., and Oldstone, M.B. 2002. Molecular anatomy of antigen-specific CD8(+) T cell engagement and synapse formation in vivo. Nat. Immunol. 3:918-925.

80. Kuhn, J.R., and Poenie, M. 2002. Dynamic polarization of the microtubule cytoskeleton during CTLmediated killing. Immunity. 16:111-121.

81. Vyas, Y.M., et al. 2001. Spatial organization of signal transduction molecules in the NK cell immune synapses during MHC class I-regulated noncytolytic and cytolytic interactions. J. Immunol. 167:4358-4367.

82. Davis, D.M., et al. 1999. The human natural killer cell immune synapse. Proc. Natl. Acad. Sci. U. S. A. 96:15062-15067.

83. Delon, J., Bercovici, N., Raposo, G., Liblau, R., and Trautmann, A. 1998. Antigen-dependent and -independent $\mathrm{Ca} 2+$ responses triggered in $\mathrm{T}$ cells by dendritic cells compared with B cells. J. Exp. Med. 188:1473-1484.

84. Dustin, M.L., and Cooper, J.A. 2000. The immunological synapse and the actin cytoskeleton: molecular hardware for T cell signaling. Nat. Immunol. 1:23-29.

85. Huppa, J.B., and Davis, M.M. 2003. T-cell-antigen recognition and the immunological synapse. Nat. Rev. Immunol. 3:973-983.

86. Trautmann, A., and Valitutti, S. 2003. The diversity of immunological synapses. Curr. Opin. Immunol. 15:249-254.

87. Davis, D.M., and Dustin, M.L. 2004. What is the importance of the immunological synapse? Trends Immunol. 25:323-327. 